\title{
An automated warehouse design validation using discrete simulation
}

\author{
Robson Peixoto ${ }^{1}$, Luís Dias ${ }^{2}$, Maria Sameiro Carvalho ${ }^{2}$, Guilherme Pereira ${ }^{2}$ and Carla A.S. Geraldes ${ }^{3}$
}

\begin{abstract}
In this paper we discuss the use of discrete simulation technique to support the redesign process of an automated warehouse system of the Brazilian Air Force unit. This unit is responsible for the supply of all uniform items and other military support material to their military forces. In particular, our work aims at analysing the inclusion of an automated sorting system to prepare uniforms individual kits in order to release downstream supply chain from this task. The main challenge was to investigate the impact on the existing storage system of a new sorting system and, simultaneously, to explore if additional management measures are required to satisfy both throughput levels and overall supply chain requirements. To accomplish a solution for such a complex problem simulation models were developed using ARENA software. Results have shown that the use of simulation allows not only the assessment of the impact of the new sorting equipment but also to test different warehouse management strategies that should be adopted concurrently to ensure that desired throughput levels can be achieved in the new scenario. In fact, this research illustrates the flexibility of the simulation tool to address several complex management issues simultaneously such as the introduction of picking sequencing rules, the use of a class-based storage policy, the addition of new picking stations, and an increase of the number of the available boxes used for transportation.
\end{abstract}

\section{INTRODUCTION}

The efficiency and effectiveness of a logistic network are largely determined by the operations that occur in their nodes (see de Koster et al. [4] and Rouwenhorst et al. [14]) Warehouses which can be considered critical nodes play a vital role in the success or failure of all the network (see Gu et al. [12]). According to Baker and Canessa [2] warehouses are also very significant from a cost perspective representing about $22 \%$ of the logistics costs in USA and about $25 \%$ in Europe. With this critical impact in the performance of the supply chain (SC) network and with high logistics costs research aimed to optimize warehouse operations has been continuously performed but is still necessary.

In modern distributions systems it is common the adoption of Automated Storage/Retrieval Systems (AS/RS) to reduce labour costs, required floor areas and error rates, while increasing products availability. However, these systems involve high investment costs and therefore require special efforts to correctly estimate the system needs. The definition of the right system configuration combined with the correct specifications and usage rules can provide optimal output rates at minimum cost investments (see Azzi et al.

${ }^{1}$ Robson Peixoto is with the Brazilian Air Force, São Paulo, Brazil (corresponding author, e-mail: robsonrtp@gmail.com).

${ }^{2}$ Luís Dias, Maria Sameiro Carvalho and Guilherme Pereira are with Centro ALGORITMI and University of Minho, Braga, Portugal (e-mail: \{lsd, sameiro, gui\}@dps.uminho.pt).

${ }^{3}$ Carla A. S. Geraldes is with the Polytechnic Institute of Bragança, Bragança, Portugal (e-mail: carlag@ipb.pt).
[1]). On the other hand, the widely implementation of information technologies (IT) such as bar coding, radio frequency communications (RFID), and warehouse management systems (WMS) provides new opportunities to improve real-time control of warehouse operations, high levels of automation and easy communication with all the different entities of the supply chain (see Gu et al. [11]).

The majority of the logistic costs that occurs in a warehouse are largely determined during the design phase. However, there are a few number of publications about the design process and there is not a comprehensive systematic method for the warehouse design problem. On the other hand, it is commonly accepted the need for evaluate the performance of the warehouse design decisions.

Performance evaluation methods may include analytical models and simulation models. According to $\mathrm{Gu}$ et al. [12] simulation is the most widely used technique for warehouse performance evaluation in the academic literature as well in practice and provide an assessment of both warehouse design and warehouse operations decisions. This is also pointed out by Geraldes et al. [10]. The authors mention that simulation can be used to validate analytical solutions and also to analyse the system behaviour, allowing the comparison of different alternatives, prior to the implementation of a particular strategy. Also for Gagliardi et al. [9] simulation techniques can help warehouse managers to evaluate the performance of warehouse operation decisions.

The scope of this work was to discuss the impact of the implementation of a new operation concerning the automated sorting process within an automated warehouse. The main purpose was to analyse if the inclusion of the new logistic operation may compromise the overall $\mathrm{SC}$ requirements or the throughput levels. To accomplish that purpose two research questions were considered:

- Q1: Are the overall requirements of the SC satisfied with the inclusion of an automated sorting system to prepare the military uniform kits?

- Q2: Are the warehouse throughput levels affected with the inclusion of the new sorting operation? This is, does this new operation affects the performance of the other existing operations within the warehouse?

The used of a simulation based approach aims also to assess the flexibility of this tool to address such a complex problem within a holistic perspective, solving simultaneously strategical and tactical or operational issues.

This paper is organized as follows. In the next section a brief literature review of the available literature on the use of simulation techniques in the automated warehouse design context is addressed. Section III describes the Brazilian Air Force uniforms supply system and section IV outlines the 
used methodology and presents all the different simulation models developed. Simulation models' results are shown and discussed in section $\mathrm{V}$ and in section VI conclusions and future work directions are mentioned.

\section{Simulation In The AUtOMATED WAREHOUSE DESIGN CONTEXT}

According to Gagliardi et al.[8] the use of simulation technique has been progressively used in the warehouse design process. Real-world systems are often too complex to be evaluated analytically and simulation models are often more precise and are quite naturally able to integrate the stochastic and the dynamic nature of the decisions. Another advantage of this technique is that it allows dealing with logistics network's upstream and downstream processes. These processes are of extreme critical importance when studying, for example, AS/RS design and control processes, and thus represent desirable research opportunities.

Automated warehouses offer an excellent opportunity for the use of simulation technique. For example, in a dualcommand cycle Storage/Retrieval (S/R) machine no mathematical model exists to analyse queuing systems with sequence-dependent service (travel) times of this type and, therefore, researchers must need to use simulation technique. Another advantage is that simulation may be used to test and better understand new systems prior to any physical implementation minimizing the risk of bad investments or failures (see Eben-Chaime et al. [6]).

According to Escobar et al. [7] the last phase of the design process of a sorting operation is the evaluation of all the subsystems in order to improve its performance. This requires an analysis of several operations processes such us: layout; storage, order picking and routing policies; and zoning.

Finally, due to advances in computer processing it is possible to use simulation techniques as a part of the warehouse design process. This technique may allow to evaluate the performance of several operations alternatives aiming to find the solutions that better meets the global supply chain requirements (see Gagliardi et al. [8]).

\section{THE BRAZILIAN AIR FORCE UNIFORMS SUPPLY SYSTEM: CASE STUDY}

The Brazilian Air Force is responsible for the supply of all uniforms and other military material to their military forces. The Supply Sub-directorate (SSD) is a Brazilian Air Force unit responsible for the forecasting, purchasing and distribution to their military personnel of all the uniforms and other support material. SSD manages two distribution centres (warehouses) located in Rio de Janeiro and São Paulo. Both distribution centres supply 36 regional warehouses that are used to store and distribute all the material to the military personnel.

After a successful experience regarding the implementation of an AS/RS in the SSD's warehouse (see Dias et al. [5]), the development of a new project concerning the replacement of both existing distribution centres by only one located in São Paulo has started. Brazilian Air Force decided to adopt a solution that includes a self-supporting automated warehouse with two aisles and two stacker cranes.
The new warehouse also includes automated guided vehicles (AGV) to fully automatic transport the products from the storage locations to the working stations.

It was also considered to change the existing warehouse design in order to include an automated sorting system. Currently, each of the 36 regional warehouses sorts specific uniform kits, tailor-made to each specific military person. The aim is to use a new automated sorting system so that all the sorting process of the uniform kits can be performed in the distribution centre instead of being performed at the regional warehouses. With this new operation it is expected an estimated reduction of about $70 \%$ in the activities of the regional warehouses, a better quality and control of the distribution process and a reduction of the stock levels in the supply chain.

\section{A. Warehouse technical features}

The initial design of the self-supporting automated warehouse includes a structure with $93 \mathrm{~m}$ length, $16 \mathrm{~m}$ width and $26 \mathrm{~m}$ height. The automated warehouse also contains eight pallet shelves (racks) accessed by single-shuttle stacker cranes in each of the two aisles. The stacker cranes (see Figure 1) have a maximum speed of $180 \mathrm{~m} / \mathrm{min}$, an acceleration of $0.8 \mathrm{~m} / \mathrm{s} 2$ ( $\mathrm{x}$ axis) and can lift a unit load with a speed of $60 \mathrm{~m} / \mathrm{min}$ and acceleration of $0.8 \mathrm{~m} / \mathrm{s} 2$ (Y axis). Also the time to store/retrieve a unit load (y axis) is 8.46 and 11.4 seconds for the first and the second depth positions respectively

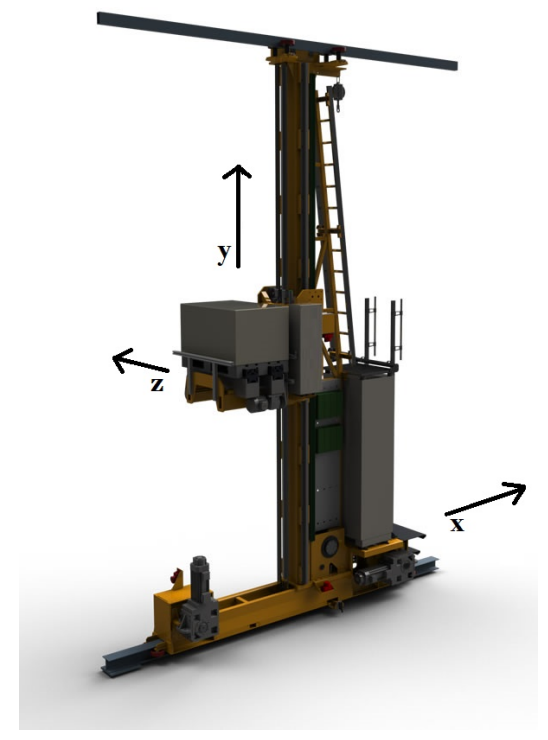

Figure 1. Single-shuttle staker crane (source: http://cassioli.com.br/produtos/).

The racks will comprise 54 horizontal sectors and 11 levels height, with a total of 4752 locations. The locations will store pallets of $1.2 \times 1.2 \mathrm{~m}$ with a load limit up to $1.5 \mathrm{~m}$ high or $1000 \mathrm{~kg}$ weight. The Input / Output (I/O) points for the aisles are locate at the bottom position near the central racks. The conveyors on the left side of each aisle are used to transport the pallets to the input points area (see Figure 2). 


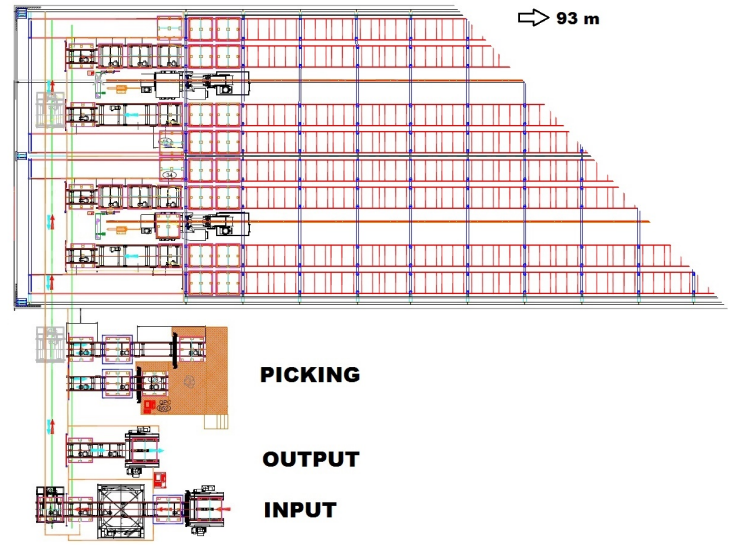

Figure 2. Scheme of the warehouse with an AS/RS system.

The other conveyors will be used to transport the pallets from the output points of the storage zone to the input point of an AGV. The AGV vehicle will transport the items between the storage and the picking area. AVG has a maximum speed of $160 \mathrm{~m} / \mathrm{min}$ and an acceleration of $0.8 \mathrm{~m} / \mathrm{s} 2$. The time to put or retrieve a unit load is 9 seconds (see Figure 3).



Figure 3. Automated guided vehicle - AVG (source: http://www.ec21.com/product-details/Rail-Guided-Vehicle--8969456.html).

\section{B. The automated picking and sorting system}

The existing automated picking system has been designed for exclusively pick the uniform kits individually, and operates in an integrated way with the AS/RS. The items picked from the shelves are placed into plastic boxes on a circular conveyor belt and then placed into the induction zone. In this area the items are removed from the boxes and displayed in the sorter. Once a uniform kit is complete it will be transported to the packing and labelling area. All the control process will be performed by radio frequency reading of electronic labels placed on pallets, storage boxes and plastic transportation boxes (see Figure 4).

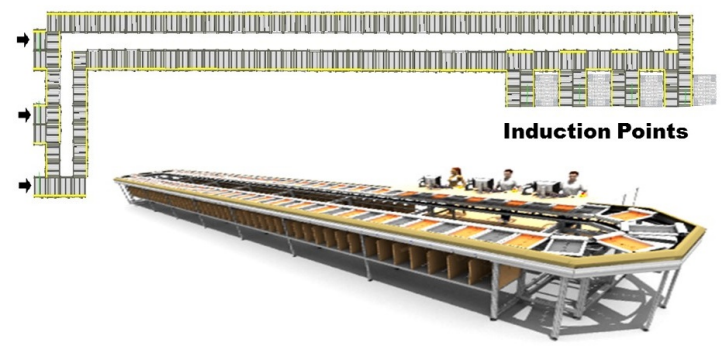

Figure 4. Sorting system (source: http://www.eurosort.com/sortersystem/index.html).

Despite the high investment cost of this new project (about 3.5 Million Euros) and its importance to the Brazilian Air Force, it is still necessary to verify if the picking operation of the uniform items jeopardizes the withdraw process of the support material, and if the project's deployment will meet the supply chain requirements. Thus, given the scarcity of available resources and due to the restricted existing area to install the system, the decision on whether to implement the picking system will depend on the estimated impact of the necessary time to prepare an individual uniform kit on the total logistic operations time of the new distribution centre. It is expected that with the implementation of the new process it will be possible to achieve a throughput level of 600 uniforms kits of about 40 to $60 \mathrm{SKU}$ each in an eight hours shift without compromising the picking activity of items not performed by the sorter.

\section{Methodology}

In order to evaluate different warehouse design strategies, several simulation models were developed using ARENA software. The aim was to validate the new warehouse design and also investigate the impact of the introduction of an automated sorting system. For that purpose, different models were developed that can be grouped in two distinct sets: (i) models without an automated sorting system; and (ii) models with an automated sorting system.

\section{A. Simulation models without an automated sorting system}

Concerning the first group of simulation models, three different models were developed. Model 1 aims to simulate the initial option of the new project that corresponds to the implementation of the AS/RS without an automated sorting system. Then, based on this initial model, two additional models were implemented with the inclusion of different operating policies. Model 2 will add a sequencing decision method based on the nearest neighbour operating policy. The nearest neighbour method consists in programming cycles of multiple commands to an automated vehicle in a way that sought items that are stored in a location close to the items to be picked. This rule looks to minimize the total transport time by minimizing travel time among locations of the items to be stored and items to be withdrawn (see Meller and Munqwattana [13]). Finally, Model 3 will be an extension of Model 2, but will include a class-based storage policy - ABC rule (see Charles et al. [3]).

All the simulation models were based on a daily working hours (simulation time of eight hours) and were programmed considering the number of working days during a month (23 repetitions). The inventory level in the warehouse was 
considered of $85 \%$ of the storage locations in each of the two storage sectors, and the time of manual picking was statistically modelled using a triangular distribution with a lower limit of 0.2 minutes, an upper limit of 1 minute, and a mode of 0.5 minutes.

Models' analysis will allow to have an estimative of the necessary time to perform in the new distribution centre all the operations currently performed in the two existing warehouses. Based on model that provides the best operational performance - Model 3- new models were developed to incorporate the new automated sorting system.

\section{B. Simulation models with an automated sorting system}

Based on Model 3, three new discrete simulation models were then developed using ARENA language (see Figure 5) to incorporate the automated sorting system considering different scenarios:

- Model 4: Model 3 including sorting operation and considering two picking stations for all the items;

- Model 5: Model 4 with the addition of two more picking stations. Two picking stations are for the exclusive use of the uniform kits preparation;

- Model 6: Model 5 with the four picking stations operating with the uniform sorting system. In this model different levels of resources (i.e. boxes) were also considered.

Based on the estimated time to prepare uniform kits (obtained from Model 3), it was assumed that picking operation is to be split in waves (slots) of two hours.

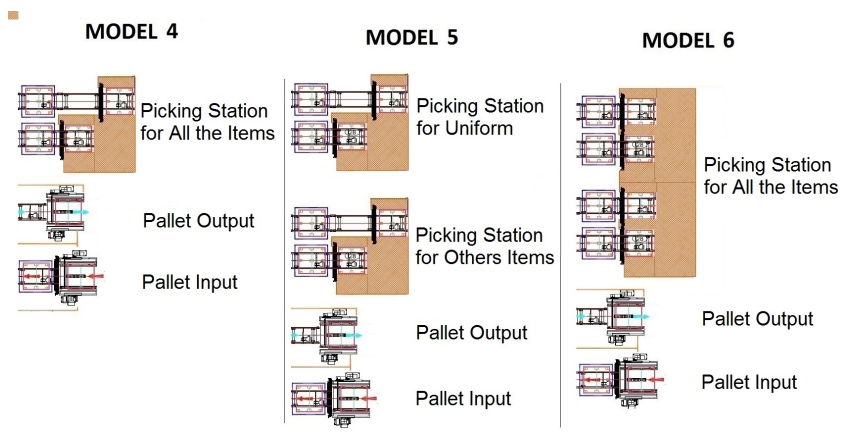

Figure 5. Picking strategies for the automated sorting system.

In order to obtain the performance indicators for the considered picking waves, all these simulation models have been programmed for a two hours' simulation time and considering 92 repetitions. The initial inventory level was also $85 \%$ of the storage locations in each of the two storage sectors of the new warehouse.

To evaluate the impact of the implementation of the automated sorting system, results from the ARENA simulation model were analysed in order to verify if the supply chain requirements were satisfied.

\section{RESUlts}

In this section we present and analyse the results of the developed simulation models. First we will show the results of the models concerning the warehouse design without the automated sorting system and then the results for the models considering the automated sorting system. These results aim to answer to the proposed research questions.

\section{A. Simulation models without an automated sorting system}

The number of movements carried out during a working day in each of first three simulated models are illustrated in Figure 6. Model 1 was developed to identify the throughput level of the existing warehouse system. Gathered results has shown that it is possible to move an average of 615 pallets during an eight hours shift. With the addition of the sequencing method based in the nearest neighbour (Model 2) it was possible to decrease the necessary time to move the items. The addition of this rule allowed an increase of about an average of 25 pallets moved during the shift, and consequently a better performance of the system. Model 3 simulation results has shown that the addition of the $\mathrm{ABC}$ classification storage policy results in a large number of picks and material movements, an average of 702 pallets.

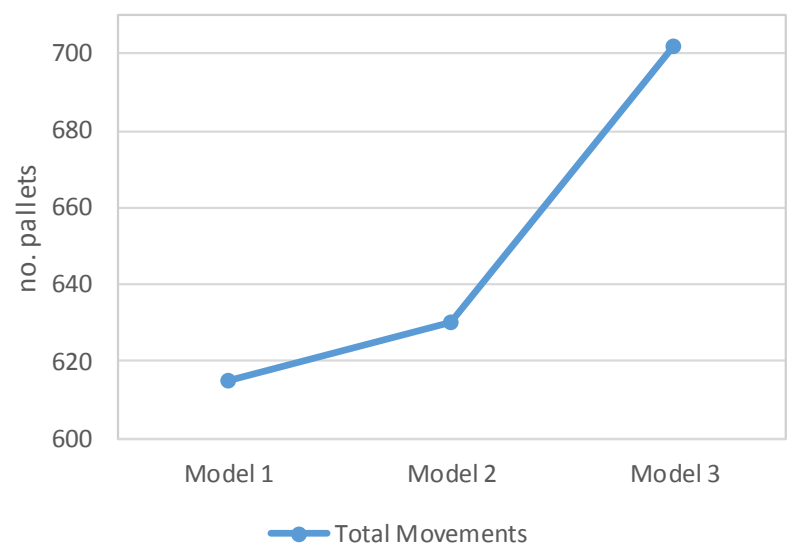

Figure 6. Productivity of the models.

As it can be seen, and taking into account the total number of storage and picking movements, the system occupancy rate of the Model 3 seems to have a better performance when compared with the two other models. This can be the result of the inclusion of the sequencing rule and the class-based storage policy. With the purpose of verify if the new system satisfies the overall requirements of the overall SC more analysis was performed considering an increase of $20 \%$ in the stock levels, according to the estimated demand for the items. The results confirmed that, once more, Model 3 presents a better performance. It was possible to conclude that the new distribution centre can replace the two existing warehouses with a reduction of $40 \%$ in the necessary time to perform the logistics operations and, at the same time, allows a decrease of $55 \%$ in the warehouse labour requirements.

\section{B. Model with an automated sorting system}

As already mentioned, Model 4 was developed based on Model 3 since it was the model that has shown the best results. Model 4 includes a sorting system and the number of picking stations were maintained. According to the results, it was possible to perform the separation of 150 uniform kits with a total of 9332 items, on every two hours. These values are consistent with a requirement of 600 uniform kits during an eight hours shift. However, as the model only has two 
picking stations, which requires two hours for the pallets processing time, it was not possible to sort the other items during the working shift. Thus, Model 5 was developed as new alternative scenario.

Model 5 included two more picking stations. This new strategy removes the products from the pallets to replenish the transportation boxes together with the storage and retrieval of the remaining items. The first two stations are dedicated to feed the sorting system and the other operate without the sorting system. However, the average of the processed items by the sorting system is smaller than the expected amount of 9000 units. As a result, a new strategy was developed in order to improve the total performance of the system.

The simulation results for Model 6 are presented in TABLE I. This model has the same number of picking stations than Model 5.

TABLE I. RESULTS OF THE SIMULATION OF MODEL 6.

\begin{tabular}{|l|c|c|c|}
\hline Parameter & Average & $\begin{array}{c}\text { Min. } \\
\text { average }\end{array}$ & $\begin{array}{c}\text { Max. } \\
\text { average }\end{array}$ \\
\hline Total pallets moved (units) & 51 & 47 & 54 \\
\hline Total boxes moved (units) & 122 & 99 & 145 \\
\hline Total items moved (units) & 9443 & 7251 & 10720 \\
\hline Occupancy rate S/R 1 (\%) & 30.08 & 22.47 & 40.68 \\
\hline Occupancy rate S/R 2 (\%) & 28.44 & 21.54 & 36.95 \\
\hline Occupancy rate AVG (\%) & 37.99 & 34.83 & 41.33 \\
\hline Occupancy rate Pickers (\%) & 40.72 & 24.39 & 69.74 \\
\hline Occupancy rate Sorter 1 (\%) & 82.17 & 62.02 & 93.29 \\
\hline Occupancy rate Sorter 2 (\%) & 81.30 & 62.88 & 92.81 \\
\hline Occupancy rate Sorter 3 (\%) & 80.35 & 63.93 & 92.32 \\
\hline Occupancy rate Sorter 4 (\%) & 79.61 & 59.47 & 91.78 \\
\hline $\begin{array}{l}\text { Total time of the operation } \\
\text { (min) }\end{array}$ & 120 & - & - \\
\hline $\begin{array}{l}\text { Total time of pallets } \\
\text { movements (min) }\end{array}$ & 113 & - & - \\
\hline
\end{tabular}

Nevertheless, in this model all the four picking stations work together with the sorting system. The aim was to verify if there is a reduction in the total sorting time and/or a decrease on the workload of the picking area. On average, it was possible to sort 9443 items by picking wave, which is more than the expected number of picks. Nonetheless, the necessary total time of 113 minutes to retrieve all the products from the pallets does not allow to perform the remaining operations concerning the other items. Consequently, low impact changes were included in this model, and new simulations were done aiming to optimize it.

We started to analyse a possible relationship between the total number of handling boxes to the buffer induction point and the necessary time to discharge the picking area. Based on the initially value of 50 boxes, six other simulations were performed with different values for the number of boxes (see Figure 7).
As it can be seen, using 55 boxes leaded to the best costbenefit ratio between the processing time of the retrieved items from the pallets and the number of boxes. However, as the result concerns to the maximum processing time of the last pallet, another simulation was performed considering a simulation time of 90 minutes. Obtained results confirmed that the average number of pallets moved is in accordance with the values shown in TABLE I.

The purpose was to evaluate if the considered time period is sufficient when the four picking stations are synchronised with the sorting system. In particular, it was necessary to verify if the time period between the handling of the last pallet and the time of the picking wave is enough to perform the retrieval of the remaining items that are not processed using the sorting system (see Figure 8).

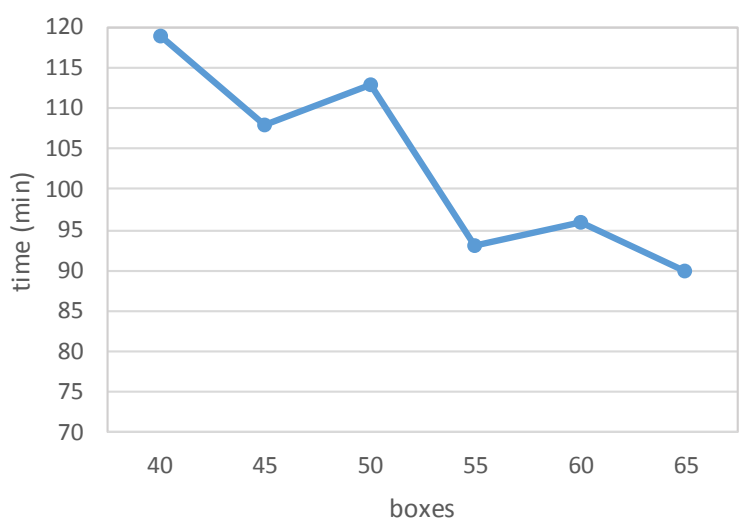

Figure 7. Pallets processing time.

Simulation results has shown that it is possible to perform a number of daily movements that exceeds the predefined values of 41 storage movements and 88 retrievals for the support items in an eight hours shift (four picking cycles) together with the uniforms sorting process.

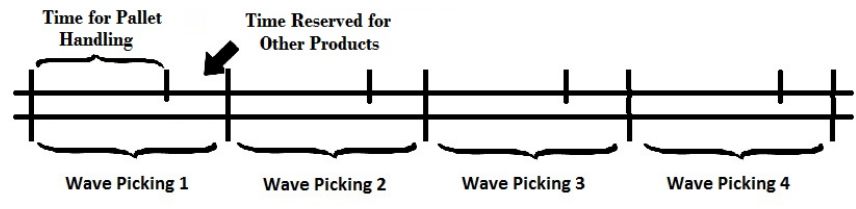

Figure 8. Handling time period scheme.

Taking into account the simulation results it was possible to conclude that the performed activities in the new warehouse system (Model 6) satisfies the overall requirements of the SC. It is then possible to replace both warehouses by the new distribution centre and also separate the uniform kits for the military persons.

\section{CONCLUSIONS AND FUtURE WORK}

In this paper we address the Brazilian Air Force warehouse redesign problem using simulation technique. A preliminary analysis of the problem identified the necessity of increasing throughput levels to cope with the extra activity of preparing uniform kits for the military forces. Therefore, in 
addition to incorporating the sorting system, additional management measures would be required to increase warehouse operations performance. An evaluation analysis using only analytical tools would be limited since it would be difficult to address simultaneously both strategic and tactical issues. However, the use of discrete simulation allows us to evaluate complex systems and to test different operating strategies prior to implementation. Furthermore, simulation models allowed a better understanding of the systems characteristics and requirements.

Data collection was performed and six simulation models were developed involving different operation scenarios:

- Model 1: initial warehouse design with the implementation of the AS/RS;

- Model 2: model 1 with a sequencing decision method based on the nearest neighbour operating policy;

- Model 3: model 2 considering a class-based storage policy;

- Model 4: model 3 with the addition of the automated sorting system;

- Model 5: model 4 with the addition of two more picking stations for the exclusive use of the uniform sorting system;

- Model 6: model 5 with the possibility of operate in two different ways: with the four picking stations operating with the uniform sorting system or using two picking stations for the uniform sorting system.

Simulation results has shown it is possible to perform all the new warehouse operations. Although it was also necessary to: (i) add sequencing rules; (ii) include storing policies; (iii) increase the number of boxes; (iv) and increase the number of picking stations to meet overall supply chain requirements.

This project can bring new insights in using simulation as a flexible tool to address several issues in a complex warehousing system and proving support in analysing different scenarios both in terms of designing and management policies. Additionally, it was also possible to analyse the performance of the warehouse considering both isolated or joint activities. Results also supports that all the operations within the warehouse are interrelated. Thus, the impact of a change in an operating policy should be verified.

Future work may include the study of the impact of batching kits uniforms in order to facilitate consolidation and shipping activities. Additionally, seasonality of demand should also be analysed and incorporated in the models, if relevant. Also important is the performance of a statistical analysis to better validate the gathered results. Finally, a return on the investment analysis can be performed. This analyses should include the number of operators that can be assigned to other activities and the inventory level reduction achieved with the centralization of the uniforms separation activity.

\section{ACKNOWLEDGMENT}

This work has been supported by COMPETE: POCI-010145-FEDER-007043 and FCT - Fundação para a Ciência e Tecnologia within the Project Scope: UID/CEC/00319/2013.

\section{REFERENCES}

[1] Azzi, A., Battini, D., Persona, A., Sgarbossa, F. and Faccio, M., "Innovative travel time model for dual-shuttle automated storage/retrieval systems", Computers and Industrial Engineering 61(3), 600-607 (2011).

[2] Baker, P., Canessa, M., "Warehouse Design: A structured approach", European Journal of Operational Research 193(2), 425-436 (2009).

[3] Charles, G. P., Gerald, R. A. and Daniel, R. H., "Improving orderpicking performance through the implementation of class-based storage", International Journal of Physical Distribution \& Logistics Management, 34(7), 534-544 (2004).

[4] de Koster, R., Le-Duc, T. and Roodbergen, K. J., "Design and control of warehouse order picking: A literature review". European Journal of Operational Research, 182(2), 481-501 (2007).

[5] Dias, R., Figueroa, H., Peixoto, R. and Sousa, L., "RFID in the central warehouse of refundable uniforms Supply Sub Directorate." Proceedings of the International Conference on RFID-Technologies and Applications (2012).

[6] Eben-Chaime, M., Pliskin, N. and Sosna, D., "An integrated architecture for simulation", Comput. Ind. Eng., 46(1), 159-170 (2004).

[7] Escobar, J., Carvalho, M. and Freires, F., "O uso de tecnologias para o processo de preparação de pedidos: implicações e proposições", Revista Produção Online, 15(1) (2015).

[8] Gagliardi, J.P., Renaud, J. and Ruiz, A., "Models for automated storage and retrieval systems: a literature review", International Journal of Production Research 50(24), 7110-7125 (2012).

[9] Gagliardi, J.P., Renaud, J. and Ruiz, A., "A simulation model to improve warehouse operations", Proceedings of the Winter Simulation Conference (2007).

[10] Geraldes, C., Carvalho, S. and Pereira, G., "Simulation in the warehouse design and management context: a survey", Proceedings of the European Simulation and Modelling Conference (2011).

[11] Gu, J., Goetschalckx, M. and McGinnis, L. F., "Research on warehouse operation: A comprehensive review", European Journal of Operational Research 177, 1-21 (2007).

[12] Gu, J., Goetschalckx, M. and McGinnis, L. F., "Research on warehouse design and performance evaluation: A comprehensive review", European Journal of Operational Research 203, 539-549 (2010).

[13] Meller, R. D. and Mungwattana, A., "Multi-shuttle automated storage/retrieval systems", IIE Transactions 29(10), 925-938 (1997).

[14] Rouwenhorst, B., Reuter, B., Stokrahm, V., van Houtum, G.,Mantel, R. and Zijm, W., "Warehouse design and control: Framework and literature review", European Journal of Operational Research 122, $515-533(2000)$ 